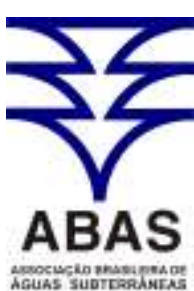

www.abas.org

\title{
GEOESTATÍSTICA E GEOQUÍMICA DOS METAIS TRAÇOS NA ÁGUA DE FONTES NATURAIS NO MUNICÍPIO DE SALVADOR - BAHIA, BRASIL
}

\author{
GEOSTATISTICAL AND GEOCHEMISTRY OF TRACE METALS IN \\ NATURAL WATER SOURCES IN THE MUNICIPALITY OF SALVADOR - \\ BAHIA, BRAZIL
}

Ana Carolina Ribeiro da Silva ${ }^{1}$, Joil José Celino², Juan Carlos Rossi Alva ${ }^{3}$, Márcio de Jesus Silva ${ }^{4}$

Artigo recebido em: 17/08/2013 e aceito para publicação em: 14/10/2013.

\begin{abstract}
This study investigates the natural and anthropogenic processes influencing the chemical composition of surface waters in Salvador (Bahia, Brazil) using multivariate statistical techniques. A total of 13 surface samples were collected between 2010 and 2011 during the rainy season, from 13 natural sources and analyzed for their physico-chemical and trace metal constituents. The chemical data generated were subjected to Principal Component Analysis (PCA) / Factor Analysis (FA) and Hierarchic Cluster Analysis (HCA). PCA is a procedure to reduce data redundancy, while FA establishes the general relationship between the variables. HCA is used to detect spatial similarity between sampling sites. The results indicate three dominant factors or proces ses for surface waters which explained $52.38 \%$ of the variance in the data set. Cluster analysis revealed a random spatial distribution of the chemical components investigated. This is consistent with the multipurpose nature of land use in the study area. The multiple natural and anthropogenic sources indicated by this study and the unsystematic distribution shows that proper land use planning and implementing existing environmental laws is critical in this region, in order to have effective management of water resources.
\end{abstract}

Keywords: Quality of surface water hydrochemistry. Principal component analysis. Factor analysis. Hierarchical cluster analysis. Polluted urban waters.

Resumo: Este estudo aborda os processos naturais e antrópicos que influenciam a composição química das águas superficiais no Município de Salvador (Bahia, Brasil), utilizando técnicas estatísticas multivariadas. Um total de 13 amostras de superfície foram coletadas entre os anos de 2010 e 2011, durante a estação chuvosa, a partir de 13 fontes naturais e analisadas por suas propriedades físico-químicas e metais traços constituintes. Os dados químicos gerados foram submetidos à Análise de Componentes Principais (ACP) / Análise Fatorial (AF) e Análise Hierárquica por Agrupamento (AHA). ACP é um procedimento para reduzir a redundância de dados, enquanto AF estabelece a relação geral entre as variáveis. AHC é usado para detectar similaridade espacial entre locais de amostragem. Os resultados indicam três processos ou fatores dominantes para as águas superficiais que explicaram $52,38 \%$ da variância no conjunto de dados. A análise de agrupamento revelou uma distribuição espacial aleatória dos componentes químicos investigados. Isto é consistente com a natureza multiuso do uso da terra na área de estudo. As múltiplas fontes naturais e antropogênicas indicadas por este estudo, e a distribuição assistemática mostra que o adequado planejamento de uso da terra e implementação das atuais leis ambientais é fundamental nesta região, a fim de ter eficaz gestão dos recursos hídricos.

Palavras-chave: Qualidade da água superficial. Hidroquímica. Análise de componentes principais. Análise fatorial. Análise de agrupamento hierárquica. Águas urbanas poluídas.

\section{INTRODUÇÃO}

Os reservatórios naturais de água vêm sendo depositários de uma variedade de subprodutos, provenientes da atividade antrópica com re percussão na saúde pública (ORTEGAGUERRERO, 2003; OMO-IRABOR et al., 2008). Avaliações superficiais e subterrâneas da qualida-

\footnotetext{
${ }^{1}$ Programa de Pós-graduação Geoquímica: Petróleo e Meio Ambiente, Universidade Federal da Bahia, Instituto de Geociências (carolinaribeiro7@yahoo.com.br).

${ }^{2}$ Programa de Pós-graduação Geoquímica: Petróleo e Meio Ambiente, Universidade Federal da Bahia, Instituto de Geociências - Bolsista de Produtividade em Pesquisa do CNPq - Nível 2 (joil@ufba.br).

${ }^{3}$ Universidade Católica do Salvador, Superintendência de Pesquisa e Pós Graduação, Laboratório de Estudos em Meio Ambiente (jcrossi@ucsal.br).

${ }^{4}$ Pós-graduação em Geociências, na Universidade Federal do Rio Grande do Sul - UFRGS (pospetro@ufba.br).
} 
de e gestão dos recursos hídricos são questões importantes que têm profundo impacto físico e químico em nossas vidas (MONTOROI, 2002; BREGUNCE et al., 2011).

A introdução de metais nos sistemas aquáticos ocorre naturalmente através de processos geoquímicos (SALOMONS \& FORSTNER, 1984) oriundos do intemperismo nas rochas percoladas e a contribuição atribuída à atividade humana é um reflexo de sua ampla utilização (CALMANO,1996; MAXIM e SPANGENBERG, 2006). Apesar de alguns deles serem essenciais aos seres vivos, como magnésio, ferro, zinco, manganês, cobre, cobalto, molibdênio e boro (atuando em diversos processos fisiológicos, ainda que em baixas concentrações), em altas concentrações geralmente podem ser tóxicos (SALOMONS \& FORSTNER, 1984; REINFELDER et al., 1998). Outros metais, como mercúrio, chumbo, cádmio, cromo e níquel, não têm função biológica conhecida e geralmente apresentam elevada toxicidade aos organismos (SALOMONS \& FORSTNER, 1984; ESTEVES, 1988).

A toxicidade, no entanto, depende de vários fatores que influenciam a disponibilidade da substância aos organismos, incluindo a fonte, a taxa de emissão, a concentração, transporte, fase de desenvolvimento e os processos bioquímicos de cada organismo (LAWS, 1993). Estas situações são comuns em reservatórios, por exemplo, devido ao aporte contínuo e excessivo de nutrientes (principalmente nitrogênio e fósforo), promovendo a eutrofização, com florescimentos constantes de algas cianofíceas e aumento da biomassa de macrófitas aquáticas (LAMPARELLI, 2004; NASCIMENTO DO VASCO et al., 2011).

Uns dos maiores exemplos da degradação ambiental dos recursos hídricos são as fontes de água naturais (PORTO et al., 1991). No entanto, a situação se torna ainda mais crítica quando os diversos metais são incorporados as fontes juntamente com os efluentes domésticos (CETESB, 2001). No Brasil, a resolução CONAMA 375/05 estabelece limites das principais propriedades físico-químicas e das concentrações de metais presentes nas águas, o que é dependente da classe na qual o corpo d'água em estudo está enquadrado (CONAMA, 2005).

A região metropolitana de Salvador (Bahia) é entrecortada e circundada pelas águas superficiais ou de canais de rios e ribeirões, com abundância de água em seu subsolo. Segundo Santos et al., (2010), “....os caminhos percorridos pelas suas águas, que recriam parte significativa da sua história, revelam o quão perversa tem sido a relação entre urbanização e natureza. As nossas águas doces desaparecem na relação inversa à intensidade do processo urbanização....".

Neste estudo, os parâmetros físicoquímicos analisados incluem o $\mathrm{pH}$, Eh, temperatura $(\mathrm{T})$, condutividade elétrica $(\mathrm{CE})$, oxigênio dissolvido (OD), nitrato $\left(\mathrm{NO}_{3}^{-}\right)$, fósforo $(\mathrm{P})$ e a extração total dos metais pesados tais como Cádmio $(\mathrm{Cd})$, Cobre $(\mathrm{Cu})$, Cromo $(\mathrm{Cr})$, Chumbo $(\mathrm{Pb})$, Ferro (Fe), Manganês (Mn), Mercúrio $(\mathrm{Hg})$, Arsênio (As) e Níquel (Ni).

Através da análise estatística multivariada, a concentração e distribuição de metais traços na água das fontes naturais no município de Salvador - Bahia foram avaliadas, comparando-as com a resolução CONAMA 375/05 para atestar sua qualidade e indicar as possíveis fontes destes metais.

\section{MATERIAIS E MÉTODOS}

A área do município de Salvador é de $693,292 \mathrm{~km}^{2}$ (IBGE, 2010), e suas coordenadas UTM, a partir do marco da fundação da cidade, no Fortaleza de Santo Antônio, são 8.565.000 norte e 551.000 leste (Fig. 1). A capital baiana conta com população de 2.675.656 habitantes (IBGE, 2010). A cidade inicialmente se instalou sobre um planalto protegido de um lado por vales profundos e do outro por uma escarpa. Atualmente o seu vetor de crescimento está direcionado ao norte - nordeste (TOURINHO, 2008).

Um total de 13 amostras foram coletadas durante a estação chuvosa à partir da água superficial de fontes naturais do município de Salvador (Fig. 1).

A lavagem dos materiais e recipientes designados à coleta e aos procedimentos analíticos ocorreu em solução de ácido nítrico $-\mathrm{HNO}_{3}^{-}$ $(10 \%)$, com subseqüente lavagem em água ultrapura (Milli-Q).

Para a realização da coleta foram utilizadas garrafas de polietileno $500 \mathrm{~mL}$ previamente aciduladas com $\mathrm{HNO}_{3}^{-}$concentrado. As amostras duplicadas foram coletadas em cada local de amostragem.

As normas e métodos de análises estão disponibilizados na tabela 1 .

Após o procedimento de coleta, as garrafas plásticas foram acondicionadas, em caixas de isopor contendo gelo $\left(-4^{\circ} \mathrm{C}\right)$ e transportadas imediatamente ao laboratório do Núcleo de Estudos Ambientais (NEA) do Departamento de Geoquímica da Universidade Federal da Bahia. 


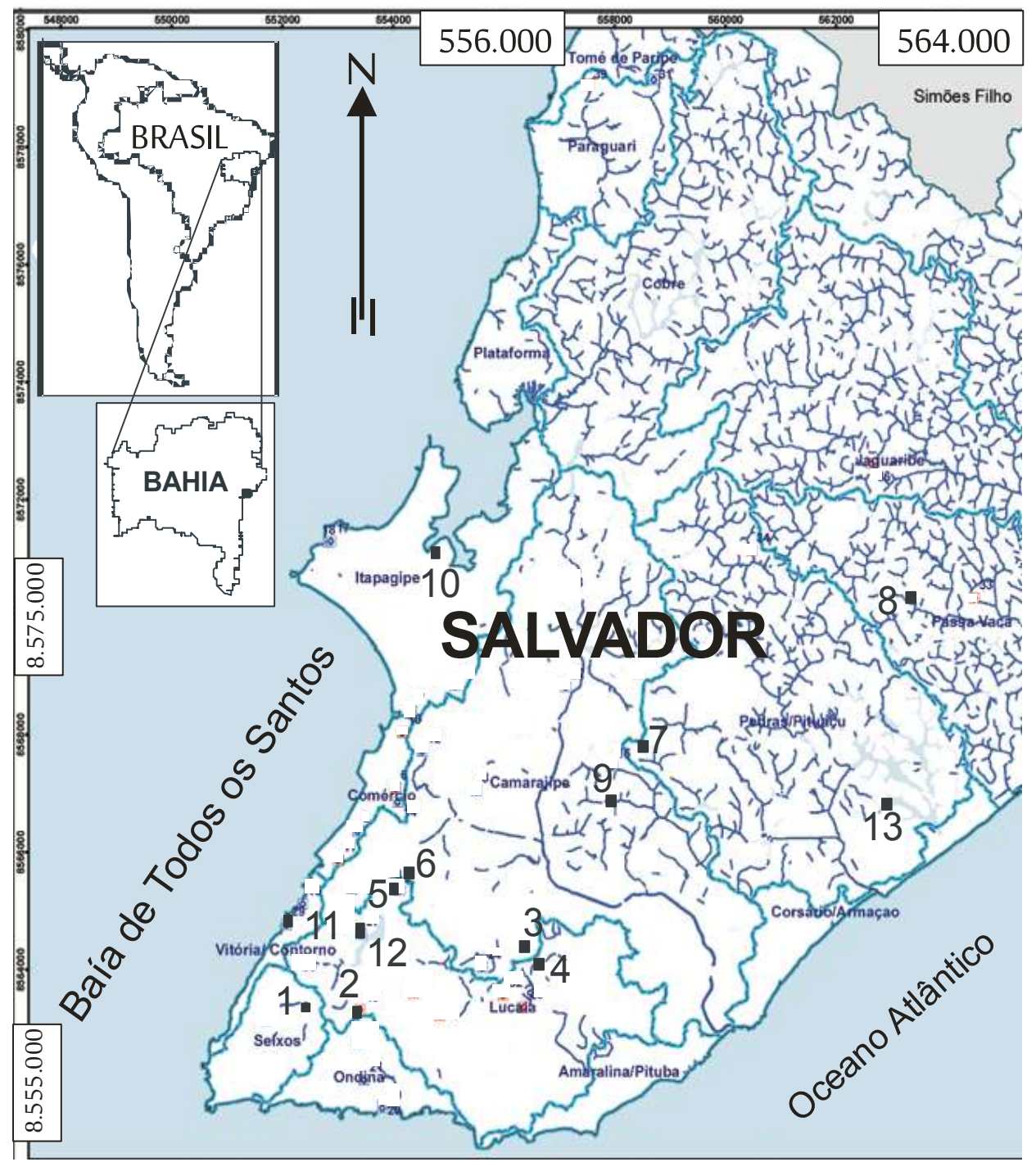

Figura 1 - Mapas de situação e localização da região metropolitana de Salvador (Bahia) com as principais fontes amostradas: (1) Pedra Furada, (2) Graça, (3) Gravatá, (4) Matatu, (5) Fonte Nova, (6) Dique, (7) Lapinha, (8) Estica, (9) Bica, (10) Santa Luzia, (11) Buraquinho, (12) Preguiça e (13) Conjunto Bahia.

Figure 1 - Situation and location map of the metropolitan region of Salvador (Bahia) with the main sources sampled: (1) Pedra Furada, (2) Graça, (3) Gravatá, (4) Matatu, (5) Fonte Nova, (6) Dique, (7) Lapinha, (8) Estica, (9) Bica, (10) Santa Luzia, (11) Buraquinho, (12) Preguiça e (13) Conjunto Bahia

Antes da execução das análises químicas, as amostras foram digeridas e tratadas com $5 \mathrm{~mL}$ de $\mathrm{HNO}_{3}^{-}$concentrado a $20 \%$ e posteriormente diluído a $2 \%$ sendo a leitura realizada em espectrometria de massas com fonte de plasma (ICPMS) para determinação dos metais $(\mathrm{Cd}, \mathrm{Cu}, \mathrm{Cr}$, $\mathrm{Pb}, \mathrm{Fe}, \mathrm{Mn}, \mathrm{Hg}$, As e Ni).

Os dados de qualidade de água foram submetidos à análise univariada: faixa de desvio, média e desvio padrão e análise multivariada: análises hierárquicas de agrupamento (AHA) e análise de componentes principais (ACP) / análise fatorial (AF). Estas análises são necessárias a uma etapa preliminar do tratamento de dados, que consistiu na normalização dos dados brutos analíticos, de modo a evitar erros de classificação, devido à ordem de magnitude e intervalo de variação dos parâmetros analíticos (ARUGA et al., 1995). Cálculos estatísticos foram executados usando o pacote de software estatístico, STATISTICA 7.0 da Statsoft Inc. 
Tabela 1 - Normas, métodos de análise e limites de quantificação (LQ)

Table 1 - Standards, methods of analysis and quantification limits

\begin{tabular}{|c|c|c|c|c|c|}
\hline Parâmetro Teste & Símbolo & Unidades & $\begin{array}{c}\text { Método do Parâmetro } \\
\text { Teste }\end{array}$ & $\begin{array}{c}\text { Descrição do } \\
\text { Método }\end{array}$ & LQ \\
\hline \multicolumn{6}{|c|}{ Parâmetro físico } \\
\hline Temperatura & Temp & ${ }^{\circ} \mathrm{C}$ & & Termômetro & 0,01 \\
\hline $\mathrm{pH}$ & $\mathrm{pH}$ & $\begin{array}{c}\text { unidades } \\
\text { de } \mathrm{pH}\end{array}$ & ASTM D1293B & medidor de $\mathrm{pH}$ & 0,01 \\
\hline Eh & Eh & $\mathrm{mV}$ & & medidor de Eh & 0,01 \\
\hline Oxigênio Dissolvido & OD & $\mathrm{mg} / \mathrm{L}$ & APHA 4500C & Iodométrico & 0,01 \\
\hline $\begin{array}{c}\text { Sólidos Totais Dissolvi- } \\
\text { dos }\end{array}$ & STD & $\mathrm{mg} / \mathrm{L}$ & APHA 208D & Medidor de STD & 0,01 \\
\hline Condutividade Elétrica & $\mathrm{CE}$ & $\mu \mathrm{s} / \mathrm{cm}$ & APHA 145 & $\begin{array}{l}\text { Medidor de Condutivida- } \\
\text { de }\end{array}$ & 0,01 \\
\hline \multicolumn{6}{|c|}{ Parâmetro químico } \\
\hline Nitrato & NO3 & $\mathrm{mg} / \mathrm{L}$ & APHA 419C & Diazotação & 0,01 \\
\hline Fósforo & $\mathrm{P}$ & $\mathrm{mg} / \mathrm{L}$ & APHA 315A & Espectro-fotômetro & 0,01 \\
\hline \multicolumn{6}{|c|}{ Metais } \\
\hline Arsênio & As & $\mathrm{mg} / \mathrm{L}$ & ASTM D2346 & ICP-MS & 0,01 \\
\hline Cádmio & $\mathrm{Cd}$ & $\mathrm{mg} / \mathrm{L}$ & ASTM D2557 & ICP-MS & 0,001 \\
\hline Chumbo & $\mathrm{Pb}$ & $\mathrm{mg} / \mathrm{L}$ & ASTM D3559 & ICP-MS & 0,01 \\
\hline Cobre & $\mathrm{Cu}$ & $\mathrm{mg} / \mathrm{L}$ & ASTM D107C & ICP-MS & 0,001 \\
\hline Ferro & $\mathrm{Fe}$ & $\mathrm{mg} / \mathrm{L}$ & ASTM D106C & ICP-MS & 0,01 \\
\hline Manganês & $\mathrm{Mn}$ & $\mathrm{mg} / \mathrm{L}$ & ASTM D108C & ICP-MS & 0,01 \\
\hline Mercúrio & $\mathrm{Hg}$ & $\mathrm{mg} / \mathrm{L}$ & ASTM D3445 & ICP-MS & 0,0001 \\
\hline Níquel & $\mathrm{Ni}$ & $\mathrm{mg} / \mathrm{L}$ & ASTM D1886 & ICP-MS & 0,001 \\
\hline
\end{tabular}

A ACP foi projetada para transformar as variáveis originais em novas variáveis não correlacionadas (eixos), chamadas de componentes principais, que são combinações lineares dos variáveis originais (SHRESTHA \& KAZAMA, 2007). Os processos que regem AF e ACP são semelhantes exceto para a preparação da matriz de correlação observada para extração e na teoria subjacente (TABACHNICK \& FIDELL, 2007). ACP preocupa-se apenas com o estabelecimento de qual componente linear existe dentro dos dados e como uma variável particular pode contribuir para o quê - propriedade física; química; etc, $\mathrm{p}$. ex. - (FIELD, 2005) do componente.

A análise fatorial (AF) produz a relação geral entre as variáveis químicas medidas, mostrando padrões multivariados que podem ajudar para classificar os dados originais (LIU et al., 2003).

O objetivo da análise de agrupamento é o de identificar grupos ou agrupamentos de dados semelhantes sobre a base de semelhanças dentro de uma classe e dissimilaridades entre diferentes classes (SPARKS, 2000). É uma técnica não supervisionada de reconhecimento de padrões que revela estrutura intrínseca ou o comportamento subjacente de um conjunto de dados sem fazer uma suposição inicial sobre os dados, a fim de classificar os objetos do sistema em categorias ou grupos com base na sua proximidade ou semelhança (PANDA et al., 2006). Agrupamento hie- rárquico aglomerativo é o método mais comumente usado em agrupamentos que são formados sequencialmente, começando com o par mais semelhante de objetos e formando aglomerado maiores passo a passo.

\section{RESULTADOS E DISCUSSÃO}

Os resultados referentes às propriedades físico-químicas e concentrações de metais na água do sistema em estudo estão apresentados na Tabela 2. Observam-se as maiores concentrações de As, $\mathrm{Fe}, \mathrm{Mn}$ e Ni, nos pontos (Fig. 2): Pedra Furada (Fonte 1), Graça (Fonte 2), Gravatá (Fonte 3), Matatu (Fonte 04), Fonte Nova (Fonte 5), Dique (Fonte 06), Estica (Fonte 8), Santa Luzia (Fonte 10), Preguiça (Fonte 12) situados na Figura 1.

Comparando-se as concentrações obtidas no presente estudo com os valores estabelecidos pela resolução CONAMA 375/05 para águas Classe 1, verifica se que as concentrações máximas obtidas nas fontes de água, pelo menos uma variável, são superiores aos valores estabelecidos pela legislação brasileira (Fig. 2). A Tabela 2 também demonstra que os valores de As, Fe, Mn e Ni foram superiores aos padrões de potabilidade para consumo humano, sendo que os metais $\mathrm{Cu}$, $\mathrm{Cd}, \mathrm{Pb}$ e $\mathrm{Hg}$ não foram detectados, em alguns pontos, em concentrações que ultrapassem esse limite (Fig. 2). 

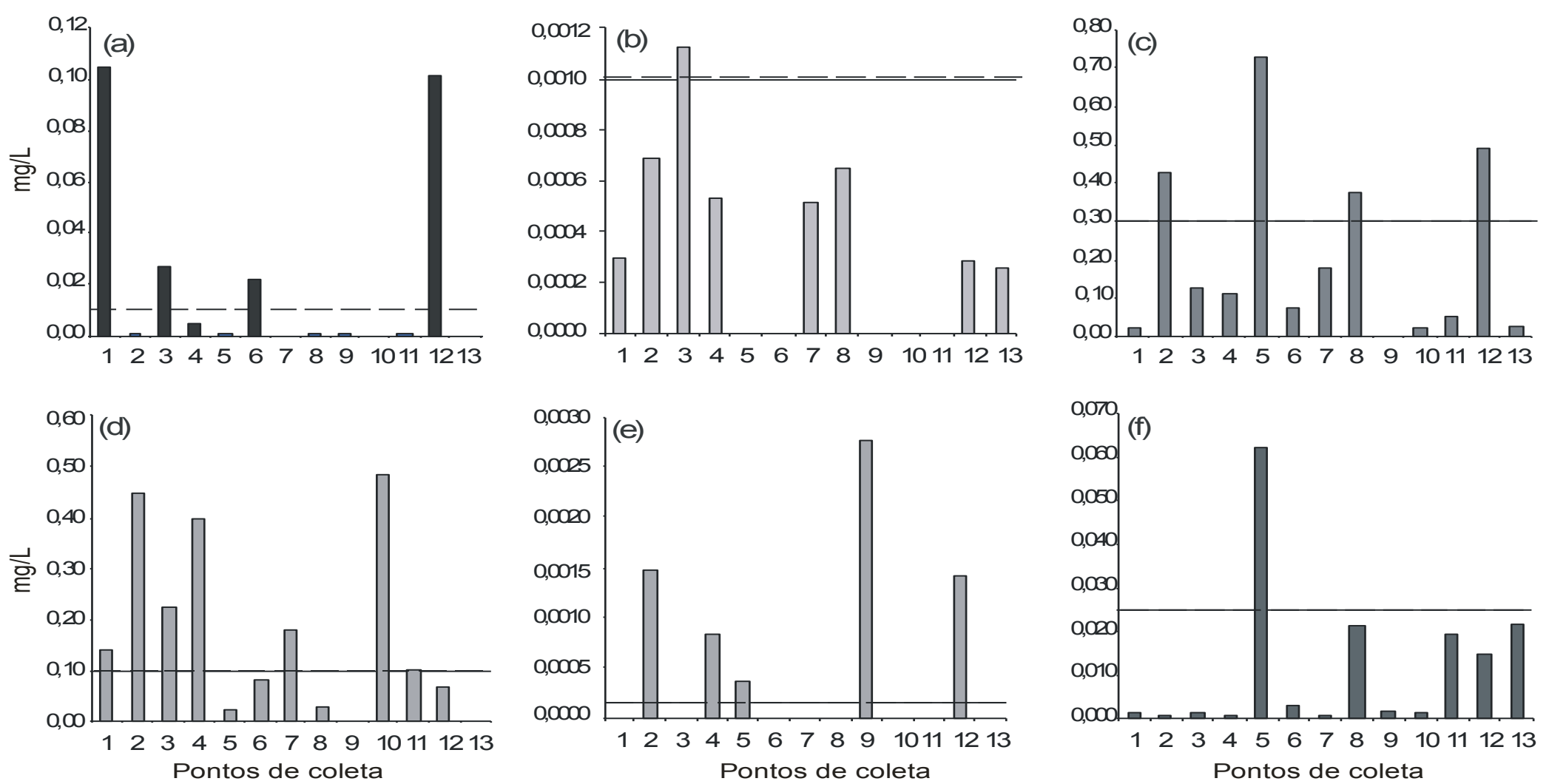

Figura 2 - Concentração (mg/L) dos metais (a) As, (b) Cd, (c) Fe, (d) Mn, (e) Hg, e (f) Ni nas fontes (vide texto para legenda) do município de Salvador (BA). A linha tracejada revela o limite máximo permitido pela portaria do CONAMA (2005)

Figure 2 - Metal concentration (mg/L) of (a) As, (b) Cd, (c) Fe, (d) Mn, (e) Hg, and (f) Ni in water sources (see text) in the Salvador city (BA). The dashed line shows the maximum allowed by ordinance of CONAMA (2005) 
Tabela 2 - Parâmetros de qualidade da água de fontes do município de Salvador (Bahia) comparados ao CONAMA (2005). Os dados em negrito apresentam valores acima do permitido

Table 2 - Quality parameters of water sources in the city of Salvador (Bahia) compared to CONAMA (2005). The data presented in bold exceed the permissible values

\begin{tabular}{|c|c|c|c|c|c|c|c|c|c|c|c|c|c|c|}
\hline Fontes & $\begin{array}{l}\text { Pedra } \\
\text { Furada }\end{array}$ & Graça & Gravatá & Matatu & $\begin{array}{l}\text { Fonte } \\
\text { Nova }\end{array}$ & Dique & Lapinha & Estica & Bica & $\begin{array}{c}\text { Santa } \\
\text { Luzia }\end{array}$ & Buraquinho & Preguiça & $\begin{array}{c}\text { Conjunto } \\
\text { Bahia } \\
\end{array}$ & \\
\hline X(UTM) & 551706 & 553255 & 553939 & 554117 & 553939 & 554524 & 555114 & 557098 & 553362 & 552827 & 552370 & 552370 & 556181 & Classe 1 \\
\hline Y(UTM) & 8563152 & 8565371 & 8565401 & 8565359 & 8564609 & 8567727 & 8568839 & 8570385 & 8566368 & 8571277 & 8565131 & 8565131 & 8568237 & CONAMA \\
\hline Pontos & $\overline{1}$ & 2 & 3 & $\overline{4}$ & 5 & 6 & 7 & 8 & 9 & 10 & 11 & 12 & 13 & $357 / 2005$ \\
\hline Temp & 24,90 & 25,90 & 25,17 & 25,00 & 25,36 & 25,13 & 24,67 & 25,91 & 26,06 & 26,08 & 25,22 & 26,36 & 25,81 & nd \\
\hline $\mathrm{pH}$ & 5,79 & 5,61 & 5,71 & 5,24 & 5,11 & 4,91 & 6,37 & 4,99 & 5,96 & 5,82 & 5,76 & 5,48 & 6,12 & 6,0 a 9,0 \\
\hline Eh & 212 & 275 & 276 & 298 & 318 & 312 & 261 & 282 & 220 & 272 & 261 & 266 & 228 & nd \\
\hline OD & 8,19 & 6,69 & 8,25 & 7,59 & 8,39 & 8,78 & 2,53 & 5,51 & 4,56 & 5,52 & 3,26 & 5,54 & 5,24 & $>6$ \\
\hline STD & 0,16 & 0,36 & 0,30 & 0,24 & 0,23 & 0,11 & 0,37 & 0,13 & 0,27 & 0,25 & 0,15 & 0,14 & 0,26 & 500 \\
\hline $\mathrm{CE}$ & 2,64 & 0,56 & 0,48 & 0,38 & 0,38 & 0,19 & 0,58 & 0,20 & 0,43 & 0,33 & 0,35 & 0,19 & 0,47 & nd \\
\hline $\mathrm{NO3}$ & 65,44 & 57,55 & 75,51 & 68,70 & $\overline{59,52}$ & 45,60 & 10,07 & 7,90 & 16,49 & 60,12 & 12,07 & 10,07 & 16,86 & 10,00 \\
\hline $\mathrm{P}$ & 10,07 & 1,68 & 1,05 & 2,08 & $\mathbf{0 , 5 0}$ & 3,75 & $\mathbf{0 , 0 8}$ & 0,20 & $\mathbf{0 , 8 2}$ & 0,76 & $\mathbf{0 , 0 8}$ & 4,06 & 1,66 & 0,10 \\
\hline As & 0,11 & 0,00 & 0,03 & 0,00 & 0,00 & 0,02 & 0,00 & 0,00 & 0,00 & 0,00 & 0,00 & 0,10 & 0,00 & 0,01 \\
\hline $\mathrm{Cd}$ & 0,000 & 0,000 & 0,001 & 0,000 & 0,000 & 0,000 & 0,000 & 0,000 & 0,000 & 0,000 & 0,000 & 0,000 & 0,000 & 0,001 \\
\hline $\mathrm{Pb}$ & 0,00 & 0,00 & 0,00 & 0,00 & 0,00 & 0,00 & 0,00 & 0,00 & 0,00 & 0,00 & 0,00 & 0,00 & 0,00 & 0,01 \\
\hline $\mathrm{Cu}$ & 0,000 & 0,008 & 0,001 & 0,010 & 0,000 & 0,000 & 0,000 & 0,000 & 0,000 & 0,000 & 0,000 & 0,009 & 0,000 & 0,009 \\
\hline $\mathrm{Fe}$ & 0,02 & $\mathbf{0 , 4 3}$ & 0,12 & 0,11 & 0,72 & 0,07 & 0,18 & $\mathbf{0 , 3 7}$ & 0,00 & 0,02 & 0,05 & 0,49 & 0,03 & 0,30 \\
\hline $\mathrm{Mn}$ & 0,14 & 0,45 & 0,22 & $\mathbf{0 , 4 0}$ & 0,02 & 0,08 & 0,18 & 0,03 & 0,00 & 0,48 & 0,10 & 0,07 & 0,00 & 0,10 \\
\hline $\mathrm{Hg}$ & 0,0000 & 0,0015 & 0,0000 & 0,0008 & 0,0004 & 0,0000 & 0,0000 & 0,0000 & 0,0028 & 0,0000 & 0,0000 & 0,0014 & 0,0000 & 0,0002 \\
\hline $\mathrm{Ni}$ & 0,001 & 0,001 & 0,001 & 0,001 & 0,062 & 0,003 & 0,001 & 0,022 & 0,002 & 0,002 & 0,019 & 0,015 & 0,022 & 0,025 \\
\hline
\end{tabular}


$\mathrm{O}$ resultado da $\mathrm{ACP} / \mathrm{AF}$ indica três fatores principais subjacentes controladores da química das águas superficiais na área de estudo (Fig. 3, Tab. 3).

O Fator 1 explica $20,47 \%$ da variância total (Fig. 3) e inclui uma carga moderada de Eh e um fraco carregamento de T e STD (Tab. 3). A forte correlação entre $\mathrm{Fe}, \mathrm{Pb}$ e $\mathrm{Ni}$ sugere uma fonte comum. Este fator pode ser atribuído às entradas em rio por escoamento superficial e os processos de erosão superficial com carreamento de partículas e elementos químicos lixiviados. As condições climáticas equatoriais e a chuva em excesso de $2000 \mathrm{~mm}$ acopladas com rochas cristalinas metamorfizadas e fraturadas, cobertas por um espesso manto regolítico e pela Formação Barreiras (NASCIMENTO \& BARBOSA, 2005), subjacente à maioria dos pontos de estudo são condições favoráveis a erosão e transporte do solo. Desta maneira, turva a água carregada de ferro lixiviado (a partir dos sedimentos lateríti$\cos$ ), $\mathrm{Pb}$ e $\mathrm{Ni}$ (a partir de lixeiras industriais e fontes agrícolas) são carregados para os rios e consequentemente para os aquíferos subterrâneos. A carga fatorial negativa de $\mathrm{OD}$ e $\mathrm{pH}$ sobre esse fator sugerem a utilização de oxigênio dissolvido na conversão ferroso para férrico acompanhada por liberação de $\mathrm{H}^{+}$para o sistema. $\mathrm{O}$ reforço do $\mathrm{Ni}$ nos rios tem sido ligada ao esgoto efluente (DASSENAKIS et al., 1998) ou fontes agrícola (MENDIGUCHÍA et al., 2007).

O Fator 2 (Fig. 3) explica 19,98\% da variância total no conjunto de dados e consiste de uma carga forte de Eh e OD e uma moderada carga de $\mathrm{NO}_{3}^{-}$e Fe (Tab. 3). Este fator pode ser atribuído a fixação biológica do nitrogênio atmosférico, da deposição a partir da atmosfera, e da intensa aplicação de fertilizantes.

O Fator 3 explica 15,38\% da variância total (Fig. 3) e consiste de uma carga forte de STD, $\mathrm{NO}_{3}^{-}$e pH (Tab. 3). A variância provavelmente representa a influência da cobertura vegetal. Seções de córrego d'água próximo às fontes que estão protegidos pela vegetação experimentam baixa temperatura e acidez também maior, resultando a partir de ácidos orgânicos derivados de folhas em decomposição que caem nos córregos. $\mathrm{O}$ efeito físico-químico do aumento do $\mathrm{pH}$ é refletido na carga negativa fraca de $\mathrm{Pb}$ sobre este fator.

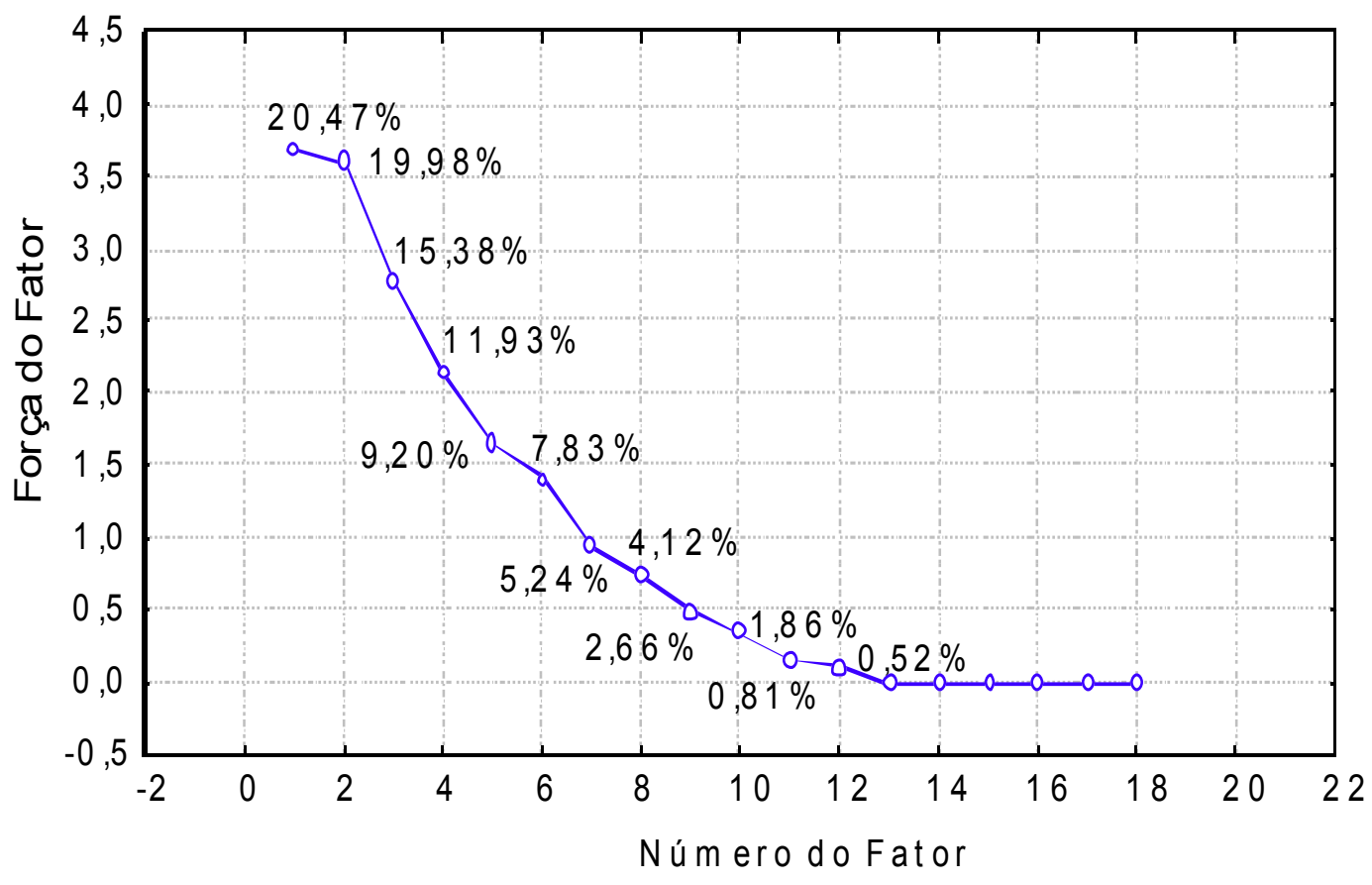

Figura 3 - Força ou "carga" de correlação dos fatores para os dados da qualidade da água das fontes naturais de Salvador (BA)

Figure 3 - Loading factor correlation for the data of water quality of natural sources of Salvador (BA) 
Tabela 3 - Matriz fatorial de carregamento varimax rotacionado para modelo de três fatores explicando $52,38 \%$ da variância total das águas superficiais das fontes. Em negrito, os valores com mais força/carga

Table 3 - Varimax rotated matrix with loading factor for three-factor model explaining 52.38\% of the total variance of surface water sources. In bold, harder values

\begin{tabular}{|c|c|c|c|}
\hline & Fator 1 & Fator 2 & Fator 3 \\
\hline Temp & 0,365 & $-0,126$ & $-0,193$ \\
\hline $\mathrm{pH}$ & $-0,037$ & $-0,812$ & 0,374 \\
\hline Eh & 0,416 & 0,815 & 0,033 \\
\hline OD & $-0,510$ & 0,715 & $-0,058$ \\
\hline STD & 0,189 & $-0,171$ & 0,797 \\
\hline $\mathrm{CE}$ & $-0,910$ & $-0,198$ & $-0,039$ \\
\hline NO3 & $-0,506$ & 0,505 & 0,409 \\
\hline $\mathrm{P}$ & $-0,916$ & $-0,002$ & $-0,227$ \\
\hline As & $-0,690$ & 0,054 & $-0,272$ \\
\hline $\mathrm{Cd}$ & $-0,130$ & 0,333 & 0,632 \\
\hline $\mathrm{Pb}$ & 0,302 & $-0,459$ & $-0,160$ \\
\hline $\mathrm{Cu}$ & $-0,056$ & 0,376 & 0,377 \\
\hline $\mathrm{Fe}$ & 0,310 & 0,678 & $-0,180$ \\
\hline $\mathrm{Mn}$ & $-0,097$ & 0,131 & 0,788 \\
\hline $\mathrm{Hg}$ & 0,089 & $-0,125$ & 0,106 \\
\hline $\mathrm{Ni}$ & 0,337 & 0,392 & $-0,602$ \\
\hline
\end{tabular}

Os 13 sítios de amostragem para a água das fontes separam em 4 grupos principais (Fig. $4)$.

O grupo I (Fig. 4) é composto por (1) Pedra Furada, (2) Graça, (3) Gravatá e (10) Santa Luzia e reflete os processos dominados pelo fator 1 .

O grupo II (Fig. 4) é constituído por (4) Matatu, (5) Fonte Nova e (6) Dique e corresponde aos locais poluídos refletido nos fatores 2 .
O grupo III (Fig. 4) está associado com escoamentos superficiais e erosão dos solos lateríticos (Fatores 1 e 2) em torno da localização (7) Lapinha, (8) Estica, (11) Buraquinho e (12) Preguiça.

O grupo IV (Fig. 4) representa a influência da poluição atmosférica e indícios de atividades vegetais no fator 3 nos locais (9) Bica e (13) Conjunto Bahia. 


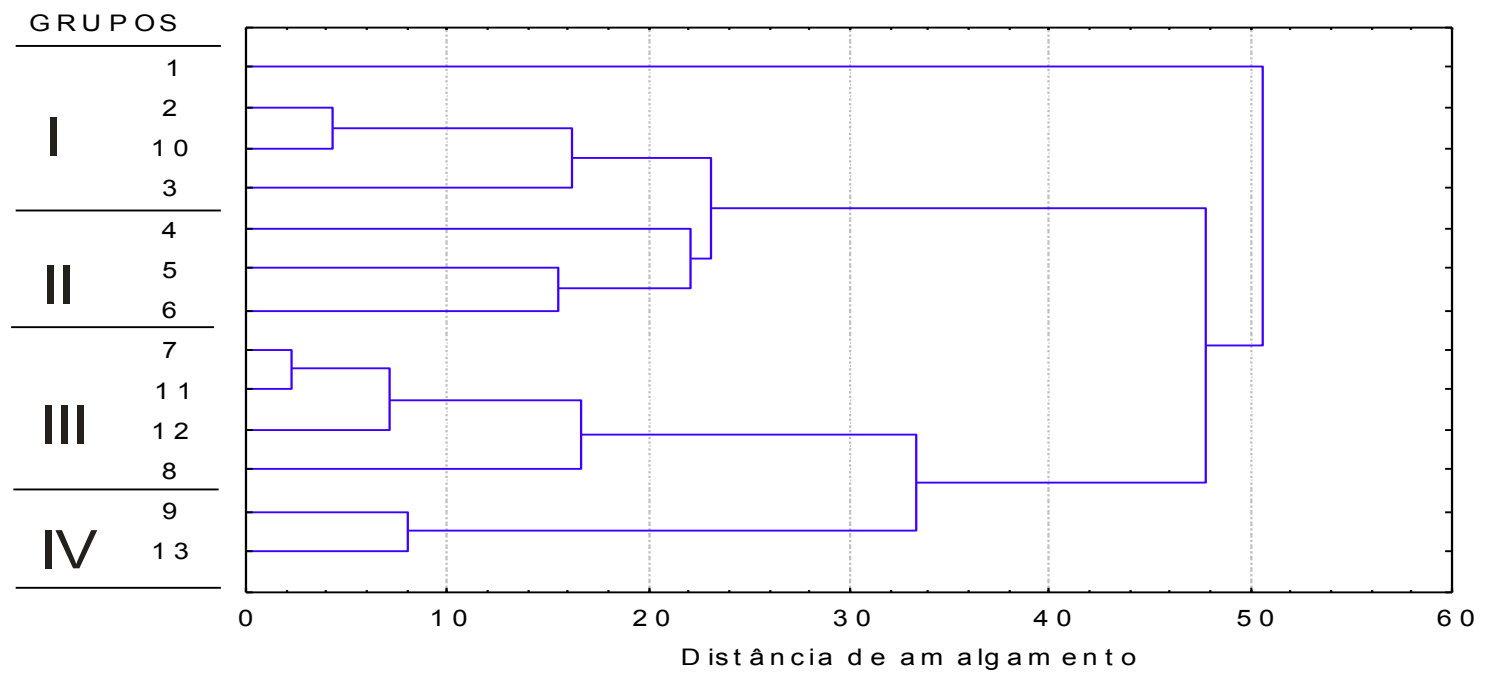

Figura 4 - Dendrograma de relacionamento entre os locais para a amostragem de águas superficiais. Cada grupo indica locais de semelhança físico-química

Figure 4 - Dendrogram between sites for surface waters sampling. Each group of local similarity indicates physical chemistry

\section{CONSIDERAÇÕES FINAIS}

Nota-se que às diversas atividades impactantes próximas as fontes analisadas culminam no transporte de metais com concentrações máximas de $\mathrm{Fe}, \mathrm{Mn}$, As e Ni, acima dos valores da Resolução CONAMA 357/2005, excedendo também aos padrões de potabilidade para consumo humano, com exceção do $\mathrm{Cu}, \mathrm{Cd}, \mathrm{Pb}$ e $\mathrm{Hg}$.

A aplicação de técnicas estatísticas multivariadas tem sido usada de forma muito eficaz para estabelecer a natureza e a distribuição espacial das variáveis físico-químicas que refletem as atividades naturais e antropogênicas em águas superficiais na região do município de Salvador (Bahia).

As seguintes conclusões foram obtidas:

Os varifatores obtidos a partir de análise fatorial para a área de estudo indicam que os processos responsáveis por variações da qualidade da água de superfície são:
- poluição industrial, doméstico e agrícola,

- atividades de erosão do solo,

- poluição atmosférica e veicular, e

- efeito vegetal.

- efeito do pH sobre o sistema.

A intensidade destes fatores naturais e antrópicos refletida na amostragem em vários locais indica uma distribuição espacial assistemática e aleatória que espelha a natureza polivalente da terra.

Estes dados demonstram que a população abastecida pelas águas das fontes naturais de Salvador, seja para consumo direto ou balneabilidade, está exposta aos metais estudados, principalmente aquela que utiliza a água sem tratamento prévio, tornando um potencial risco à saúde. Portanto, o planejamento do uso adequado do solo é imperativo na região estudada para uma eficaz gestão e monitoramento das águas superficiais.

\section{AGRADECIMENTOS}

O primeiro autor agradece à CAPES/FAPESB pela concessão de bolsa de pesquisa. Ao Instituto de Química (UFBA) pelas análises químicas, assim como à equipe do NEA e do Pospetro da Universidade Federal da Bahia, pela ajuda nos trabalhos de campo.

\section{REFERÊNCIAS}

APHA, AWWA, WPCF. Standard methods for the examination of water and wastewater. 20. ed. Washington D. C. /USA, American Public Health Association, 2000.
ARUGA, R., GASTALDI, D., NEGRO, G., OSTACOLI, G. Pollution of a river basin and its evolution with time studied by multivariate 
statistical analysis. Analytica Chimica Acta. v. 310, p. 15-25. 1995.

ASTM. American Society for Testing and Materials Standards. New York/USA, American Standard for Testing Materials, 2001.

BREGUNCE, D. T.; JORDAN, E N.; DZIEDZIC, M.; MARANHO, L. T.; CUBAS, S. A. Avaliação da Qualidade da Água do Ribeirão dos Müller, Curitiba-PR. RBRH - Revista Brasileira de Recursos Hídricos. v. 16, n. 3, p. 39-47. 2011.

CALMANO, W. Metals in sediments: Remobilization and environment hazards. In: MUNAWAR, M. \& DAVE, G. (eds.) Development and Progress in Sediment Quality Assessment: Rationale, Challenges, Techiques \& Strategies. 1996. 272p.

CETESB. Qualidade das águas no Estado de São Paulo: águas e energia elétrica. Companhia De Tecnologia De Saneamento Ambiental. São Paulo, n. 14, p.11-22. 2001.

CONAMA 375. Resolução CONAMA 20 de 12 de junho de 2005. Conselho Nacional do Meio Ambiente. 2005.2 Disponível: http://www.cetesb.sp.gov.br/Agua/praias/res_cona ma 357 05.pdf. Acesso em março, 2012.

DASSENAKIS, M.; SCOULLOS, M.; KRASAKOPOULOU, E.; PLAVIDOU, A.; KLOUKINIOTOU, M. Effects of multiple source pollution on a small Mediterranean river. Applied Geochemistry. v. 13, p. 197-211. 1998.

ESTEVES, F. A. Fundamentos de limnologia. Rio de Janeiro, Interciência, FINEP. 1988. 575p.

FIELD, A.P. Discovering Statistics Using SPSS: And Sex, Drugs and Rock ' $n$ ' Roll. Sage Publications, London/ENG. Report.. 2005.

IBGE. Instituto Brasileiro de Geografia e Estatística. 2010. http://www.ibge.gov.br/cidadesat/topwindow.htm ?1. Acesso em março, 2012.

LAMPARELLI, M. C. Graus de trofia em corpos d'água do estado de São Paulo: avaliação dos métodos de monitoramento. Tese de Doutorado. Instituto de Biociências da Universidade de São Paulo. 2004. 150p. Disponível em: http://www.teses.usp.br/teses/disponiveis/41/4113 4/tde-20032006-

075813/publico/TeseLamparelli2004.pdf. Acesso em março, 2012.
LAWS, E. A. Aquatic pollution: an introductory text.. 2. ed. Interscience publication, John Wiley \& Sons, INC. New York/USA, 1993, 482p.

LIU, C.-W.; LIN, K.-H.; KUO, Y.-M. Application of factor analysis in the assessment of groundwater quality in a blackfoot disease area in Taiwan. Science of the Total Environment. v. 313, p. 77-89. 2003.

MAXIM, L., SPANGENBERG, J.H., Bridging the gap between two analytical frameworks. Ninth Biennial Conference of the International Society for Ecological Economics. New Delhi, India. 2006. Disponível em http://seri.academia.edu/JoachimHSpangenberg/P apers/300783/An Analysis of Risks for Biodiv ersity Under the DPSIR Framework. Acesso em março, 2012.

MENDIGUCHIA, C.; MORENO, C.; GARCIAVARGAS, M. Evaluation of natural and anthropogenic influences on the Guadalquivir River (Spain) by dissolved heavy metals and nutrients. Chemosphere, Corrected Proof, p. 3439. 2007.

MONTOROI, J-P; GRÜNBERGER, O.; NASRIB, S. Groundwater geochemistry of a small reservoir catchment in Central Tunisia. Applied Geochemistry , v. 17, p. 1047-1060. 2002.

NASCIMENTO, S. A. M. \& BARBOSA, J. S. Qualidade da Água do Aquífero Freático no Alto Cristalino de Salvador, Bacia do Rio Lucaia, Salvador, Bahia. Revista Brasileira de Geociências, v. 35, n. 4, p. 543-550, 2005.

NASCIMENTO DO VASCO, A.; BRANDÃO BRITTO, F.; SOUSA PEREIRA, A. P., VIEIRA MÉLLO JÚNIOR, A.; BORGES GARCIA, C. A.; NOGUEIRA, L. C. Avaliação espacial e temporal da qualidade da água na sub-bacia do rio Poxim, Sergipe, Brasil. Ambiente \& Água. 2011. Disponível em: $<$ http://redalyc.uaemex.mx/src/inicio/ArtPdfRed.j sp? $\mathrm{iCve}=92817183010>\quad$ ISSN $\quad 1980-993 \mathrm{X}>$ Acesso em março, 2012.

OMO-IRABOR, O. O; OLOBANIYI, S. B.; ODUYEMI , K.; AKUNNA, J. Surface and groundwater water quality assessment using multivariate analytical methods: A case study of the Western Niger Delta, Nigeria. Physics and Chemistry of the Earth, v. 33, p. 666-673. 2008. 
ORTEGA-GUERRERO, A. Origin and geochemical evolution of groundwater in a closed-basin clayey aquitard, Northern Mexico. Journal of Hydrology, v. 284, p. 26-44. 2003.

PANDA, U. C.; SUNDARAY, S. K.; RATH, P.; NAYAK, B. B.; BHATTA, D. Application of factor and cluster analysis for characterization of river and estuarine water systems - a case study: Mahanadi River (India). Journal of Hydrology, v. 331, p. 434-445. 2006.

PORTO, M.F.A.; BRANCO S.M.; DE LUCA S.; J. Caracterização da qualidade das águas. In: Branco, S. M. \& PORTO, R. L. L. (eds.) Hidrologia Ambiental. Coleção ABRH de Recursos Hídricos, EDUSP, São Paulo, 1991, 411p.

REINFELDER, J. R.; FISHER, N. S.; LUOMA, S. N.; NICHOLS, J. W.; WANG, W. X. Trace element trophic tranfer in aquatic organisms: a critique of the kinetic model aproach. The Science of the Total Envirnonment., v. 219, p. 4553. 1998.

SALOMONS, W. \& FÖRSTNER, U. Metals in the hydrocycle. Springer-Verlag. 1984, 349p.
SANTOS, E. et al. O Caminho das Águas em Salvador: Bacia Hidrográficas, Bairros e Fontes. Centro Interdisciplinar de Desenvolvimento e Gestão Social - CIAGS da EA-UFBA. Disponível em: $<$ http://www.gestaosocial.org.br/almanaque-dasaguas $>$ Acesso em novembro 2010.

SHRESTHA, S. \& KAZAMA, F. Assessment of surface water quality using multivariate statistical techniques: a case study of the Fuji river basin, Japan. Environmental Modelling \& Software. v. 22, p. 464-475. 2007.

SPARKS, T. Statistics in Ecotoxicology. John \& Wiley Ltd, Chichester. 2000.

TABACHNICK, B. G.; FIDELL, L. S. Using Multivariate Statistics. Pearson/Allyn \& Bacon, London/ENG. $5^{\text {a. }}$ ed. 2007. 252p.

TOURINHO, A. O. Estudo histórico e sócio ambiental das principais fontes públicas de Salvador. Dissertação de Mestrado - Universidade Federal da Bahia. UFBA. 2008. 155p. 